\title{
THE EFFECTS OF DISTRIBUTED CAPACITY OF COILS USED IN RADIO-TELEGRAPHIC CIRCUITS.
}

\author{
By Frederick A. Kolster \\ (United States Bureau of Standards)
}

During the past eight or ten years much more attention has apparently been given to the development of the transmitting apparatus in radio-telegraphy than to the receiving apparatus.

Consequently the same sort of apparatus used five years ago or more to receive highly damped multi-frequency waves is still in use to receive the more persistent waves of single frequency which result from the improved transmitters of to-day.

It is, of course, apparent that the more nearly undamped are the transmitted waves, the more care must be taken in the design of the receiving apparatus.

Among the many things to consider in the design of circuits in which persistent radio frequency currents exist, are the effects of distributed capacity in the coils of the circuit.

It is the purpose of this paper to give the results of some preliminary experiments which were conducted with the view of determining how important are the effects of the distributed capacity of coils used in radio frequency circuits. At first thought, these effects would seem to be of minor importance, and this is generally true if proper precautions are taken in the design of the circuits and the construction of the coils.

In much of the apparatus in practical use, however, those precautions have apparently not been taken, with the result that peculiar phenomena are often observed. In laboratory apparatus for calibration and measuring purposes consideration of distributed capacity effects is of extreme importance.

It is not unusual in practise to find that coils used in wave meters and receiving apparatus have distinct natural periods or frequencies in the range of frequencies for which the circuit may be adjusted. 
If the inductance of such a coil is measured at various frequencies, it will be found that this inductance apparently changes with the frequency as shown by the curve of Figure 1. The crosses on the curve indicate experimentally observed values. The natural wave length of the coil tested is about 260 meters and its inductance approximately 1.5 milli-henrys when measured at very low frequencies.

If it is assumed that a coil having distributed capacity may be, for all practical purposes, artificially represented by a loop circuit containing inductance and capacity in parallel as shown in the diagram of Figure 1, then we may write for the apparent inductance of the coil, if resistance is neglected,

$$
\mathrm{L}^{1}=\frac{\mathrm{L}}{1-\mathrm{L} \mathrm{C}^{1} \omega^{2}}
$$

where $\mathrm{L}$ is the true inductance of the coil, $\mathrm{C}$ its effective capacity, and $\omega$ equals $2 \pi$ times the frequency.

If this assumption is good, it should be possible to determine the values of $\mathrm{L}$ and $\mathrm{C}$ of an equivalent loop circuit such as to make its apparent inductance equal to that of the coil under test for the range of wave lengths desired.

Considering now the curve in Figure 1, we may write for any two wave length or their corresponding values of $\omega$,

$$
L_{11}^{1}=\frac{\mathrm{L}}{1-\mathrm{LC} \omega_{1}^{2}} \text { and } \mathrm{L}_{2}^{1}=\frac{\mathrm{L}}{1-\mathrm{L} \mathrm{C} \omega_{2}^{2}}
$$

By elimination we get

$$
L=\frac{L_{1}^{1} L_{2}^{1}\left(\omega_{1}^{2}-\omega_{2}^{2}\right)}{L_{1}^{1} \omega_{1}^{2}-L_{2}^{1} \omega_{2}^{2}}
$$

or in terms of wave lengths, since $\omega=\frac{2 \pi \mathrm{v}}{\lambda}$

$$
C=\frac{L_{1}^{1} L_{2}^{1}\left(\lambda_{2}^{2}-\lambda_{1}^{2}\right)}{L_{1}^{1} \lambda_{2}^{2}-L_{2}^{1} \lambda_{1}^{2}}
$$

Averaging the results obtained by taking several pairs of points on the curve in Figure 1, a value of 1.50 millihenrys is obtained for the true inductance of the coil measured.

The value of the effective capacity of the coil may be 

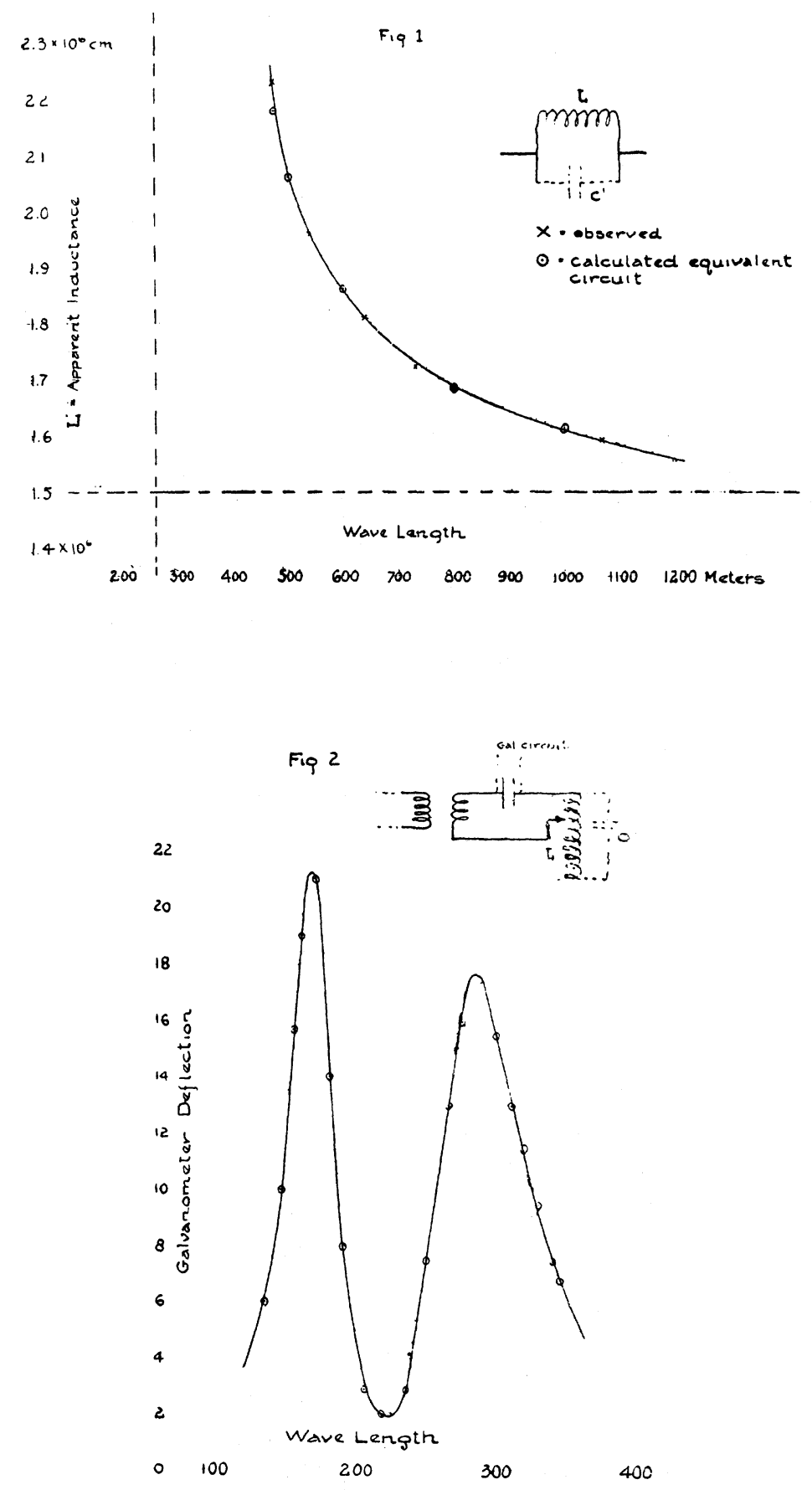
expressed as

$$
\mathrm{C}=\frac{1-\frac{\mathrm{L}}{\mathrm{L}^{1}}}{\mathrm{~L} \omega^{2}}
$$

or, in terms of the wave lengths as

$$
\mathrm{C}=\frac{\lambda^{2}}{4 \pi^{2} \mathrm{v}^{2}}\left(\frac{1}{\mathrm{~L}}-\frac{1}{\mathrm{~L}^{1}}\right)
$$

Averaging the results obtained for various wave lengths we get for this capacity, 0.000013 micro-farads.

The coil in question may, therefore, be represented by an equivalent loop circuit having a condenser of 0.000013 microfarad in parallel with a coil of 1.50 millihenry. The circles on the curve in Figure 1 indicate the calculated apparent inductance of this equivalent circuit for the ranges of wave lengths indicated, showing that the assumption made is good for all practical purposes.

The diagram shown in Figure 2 represents a common form of circuit used in receiving apparatus and in some forms of wave meters. The loading coil $\mathrm{L}$ of considerable inductance and distributed capacity is divided into sections one or more of which may be connected in circuit to allow adjustment for various ranges of wave lengths. The imaginary condenser in parallel with this coil represents the effective capacity of the coil. For short wave lengths only part of the coil is in circuit, the unused sections being, however, inductively related to the part in circuit. It will be seen that this arrangement is really a case of two closely coupled circuits which should undoubtedly respond to two distinct frequencies or wave lengths. This fact is shown to be true by the experimentally obtained curve in Figure 2.

A brief theoretical consideration of a circuit of two degrees of freedom as shown in Figure 2 is of interest. This circuit may be diagrammatically represented by the circuit in Figure 3.

The reactance of the loop circuit $11^{1} c^{1}$, if resistance be neglected, may be written,

$$
\mathrm{X}=\frac{1 \omega\left(1^{1} \mathrm{C}^{1} \omega^{2}-1\right)}{\left(1^{1}+1\right) \mathrm{C}^{1} \omega^{2}-1}
$$

The curve (a) in Figure 3 shows the variation of the reactance of such a loop circuit for various values of $\omega$. Curve 

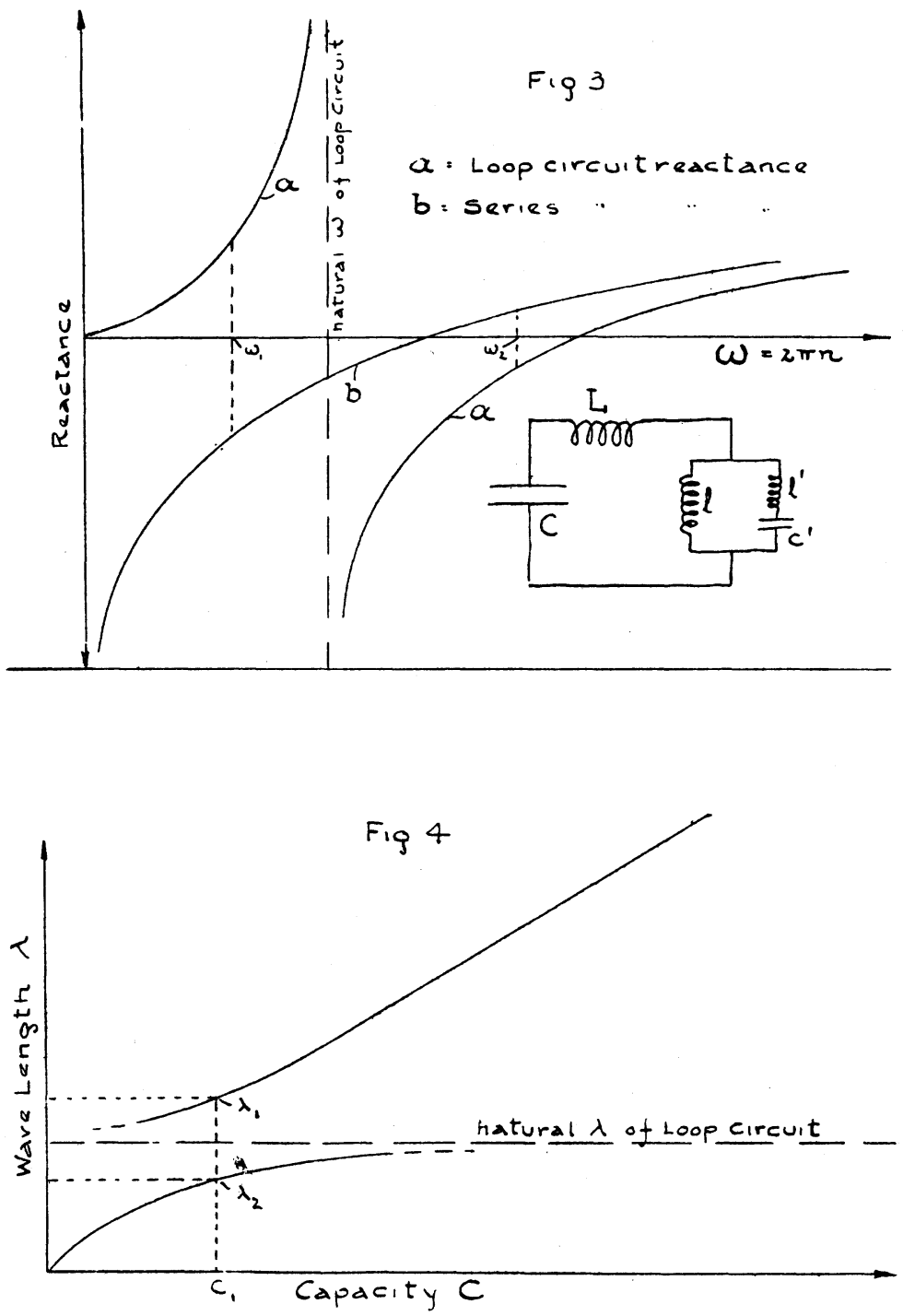
(b) gives the reactance of the series circuit L,C for various values of $\omega$.

The total reactance of the system for various values of $\omega$ is therefore the sum of these two curves, and it is seen that zero reactance is obtained for two values of $\omega$, showing that for any particular setting of the condenser $C$, the system will respond to two distinct wave lengths.

For various settings of the condenser $\mathrm{C}$, a calibration curve as shown in Figure 4 is obtained. Such cases as this actually occur in practice, the cause being entirely due to the effects of distributed capacity in coils.

The radio-frequency resistance of coils with appreciable distributed capacity is found in practice to be higher for some frequencies than that calculated from the well-known formulae. This may be at least partly explained if we again assume that such coils may be represented by equivalent loop circuits.

A loop circuit having an inductance $\mathrm{L}$ with calculated radio-frequency resistance $R$, in parallel with a condenser $C$ as shown in the diagram of Figure 7 will have an apparent resistance

$$
\mathrm{R}^{1}=\frac{\mathrm{R}}{\mathrm{R}^{2} \mathrm{C}^{2} \omega^{2}+\left(\mathrm{L} \mathrm{C \omega ^{2 }}-1\right)^{2}}
$$

Curve (a) in Figure 7 gives the calculated radio-frequency resistance of a particular coil for various wave lengths. Curve (b) gives the apparent radio frequency resistance of this coil for this range of wave lengths, taking into consideration the distributed capacity and treating it as a loop circuit.

Distributed capacity effects in the coils of the so-called untuned or aperiodic detector circuits are in many cases very striking, the result being that the circuit is not at all aperiodic but responds much more violently at a particular frequency depending upon the natural period of the coil.

The so-called untuned detector circuit is shown in the diagram of Figure 5. The curve in this figure shows experimentally observed galvanometer deflections when the circuit is excited at various wave lengths. A comparatively sharp resonance curve is obtained showing that the circuit is distinctively not aperiodic.

An extremely interesting and ingenious method for determining the value of the effective capacity of a coil is described 
Fig 7

$22^{\Omega}$

20

18

16

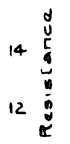

10

8

6 a - calculated resistance b - apparent

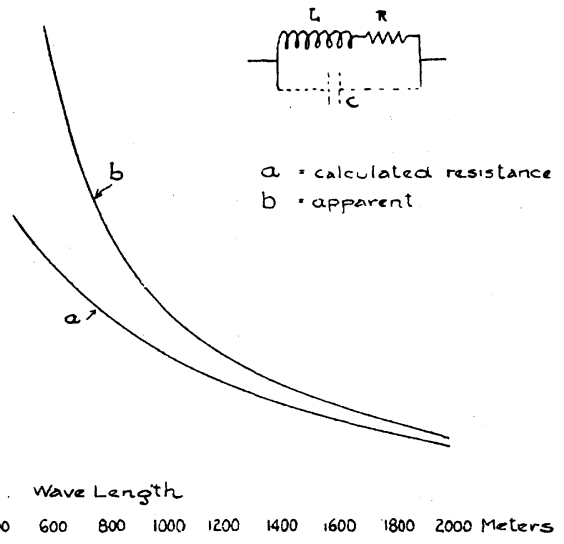

$\begin{array}{llllllllll}200 & 400 \quad 600 \quad 800 \quad 1000 \quad 1200 \quad 1400 \quad 1600 & 1800 & 2000 \text { Meters }\end{array}$

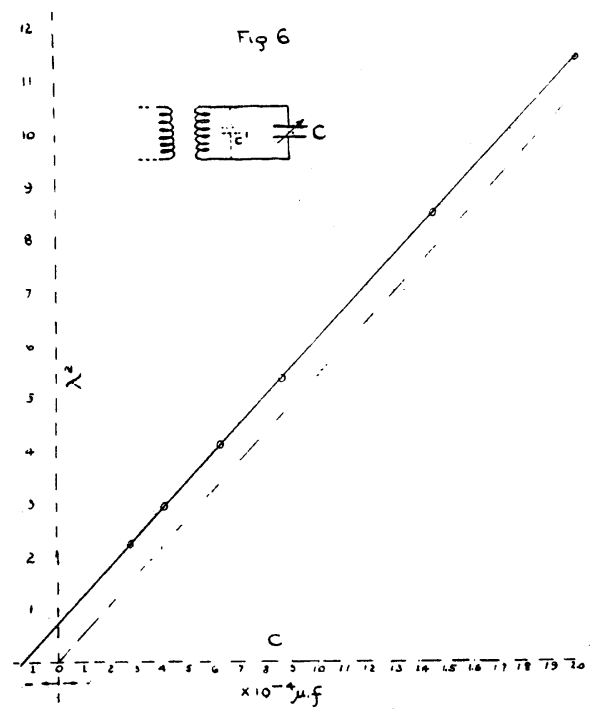

12

Fig 5
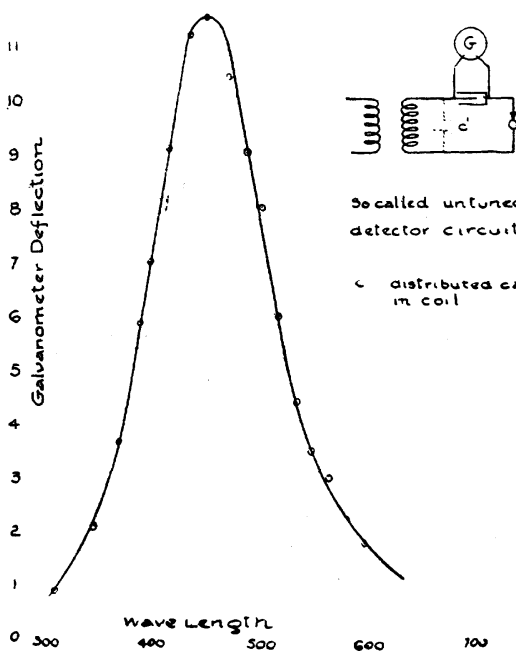

So called untuned detector circuit

distributed capacity
in coil

$400 \quad 500 \quad 600 \quad 100$ 
in an article written by Professor G. W. O. Howe in the Proceedings of the Physical Society of London.

A variable condenser of known capacity is connected to the coil under observation and the circuit is excited at various frequencies. A curve as shown in Figure 6 is plotted with values of condenser capacity as abscissae and the squares of the wave lengths as ordinates. A straight line is obtained which, instead of striking the horizontal axis at zero, which would be the case if no distributed capacity existed in the coil, gives the negative value of $\mathrm{C}$ corresponding to a value zero for the square of the wave length. This negative value is the effective capacity of the coil.

The true value of the inductance of the coil may also be determined from the broken line shown in Figure 6, drawn parallel to the observed line, through $\mathrm{C}$ equal to zero. Taking value of $\lambda^{2}$ as determined by this broken line for various values of $\mathrm{C}$, and averaging the results from the usual formulae

$$
\mathrm{L}=\frac{\lambda^{2}}{4 \pi^{2} \mathrm{v}^{2} \mathrm{C}}
$$

the value of the true inductance is obtained.

The curve in Figure 6 was obtained for the same coil as in Figure 1, and the values for the effective capacity and true inductance of this coil as determined by these two methods agree almost exactly.

The results of these experiments show the importance of taking into consideration the capacity effects in coils of circuits designed for calibration and standardizing purposes, and in particular in circuits of large inductance and small capacity.

Inductance coils for radio-frequency circuits should be designed to have minimum capacity as well as minimum resistance. It is unfortunate that the best design for one of these requirements is generally not the best design for the other.

Coils with "dead-ended" turns should not be used, even though the turns not in use are metallically disconnected from the circuit. They should be entirely out of the field of the active turns.

Coils in so-called untuned detector circuits should be particularly designed to have minimum capacity or else for each short range of wave lengths a separate coil should be used having a natural period best adapted to this range.

SUMMARY: It is shown experimentally that an induc- 
tance having distributed capacity may be practically replaced by an inductance (called its "true inductance") in parallel with a capacity, (called its "effective capacity"). Methods of calculating each from observations are given and it is shown how strong response to one or more frequencies in wave meter and so-called untuned detector circuits is caused by distributed capacity inductances, particularly those with dead-ends. Practical conclusions are drawn.

\section{DISCUSSION.}

ALFRED N. GOLDSMITH: The assumption made by $\mathrm{Mr}$. Kolster that an inductance having distributed capacity may be for electrical purposes replaced by a definite inductance in parallel with a definite capacity can be true only when the inductance is reasonably localized and the capacity not extremely great. It certainly cannot hold in unmodified form for radiative antennae. By experiment it has been shown to be a valid assumption (within the errors of measurement) for practically non-radiative coils of moderate dimensions.

An interesting fact concerning an open or radiative circuit, that is, one containing large distributed capacity, is that the frequencies of the alternating currents produced in such a circuit when it is coupled to a closed oscillating circuit cannot be correctly calculated from the equations holding for closed coupled circuits, and that the frequencies of the overtones produced in the open circuit are not integral multiples of the fundamental frequency. (See L. Cohen, Bulletin Bureau of Standards, Vol. VI, No. 2, 1909).

GREENLEAF W. PICKARD: Distributed capacity effects in inductances are important radio apparatus, and are usually detrimental. While it is obvious that such capacity cannot be entirely eliminated, it may be minimized in various ways. Multiple layer coils of ordinary construction have excessive distributed capacity, and many more or less successful attempts have been made to decrease this. Certain early patents in this art show multiple layer coils, wound in a peculiar manner, each section of the coil having its layers 